\title{
IMPLICAÇÕES NA QUALIDADE DO ATENDIMENTO CIRÚRGICO DIANTE DA NÃO MANUTENÇÃO DOS EQUIPAMENTOS HOSPITALARES
}

\author{
Implications for the quality of surgical care through \\ the non-maintenance of hospital equipment
Implicaciones para la calidad de la atención quirúrgica por medio del no mantenimiento de los equipos hospitalarios

Thabata Coaglio Lucas ${ }^{1 *}$, Anita Cassia Aguiar Reis², Pâmela Peçanha de Moraes ${ }^{3}$, Dulce Aparecida Martins ${ }^{4}$

RESUMO: Objetivo: Identificar as implicações da não manutenção dos equipamentos hospitalares na qualidade do atendimento cirúrgico. Método: Trata-se de uma pesquisa quantitativa, exploratória, descritiva, observacional, realizada em um hospital filantrópico do interior de Minas Gerais. Aplicou-se a técnica de observação direta e a avaliação de registros de manutenção preventiva e corretiva dos equipamentos cirúrgicos. Resultados: Durante o período de observação, verificou-se que os equipamentos que mais apresentaram falhas durante a cirurgia foram: bisturi elétrico, intensificador e foco cirúrgico. Os dados de funcionalidade e manutenção dos equipamentos foram comparados com recomendações do fabricante e com a literatura científica. Conclusão: A não manutenção dos equipamentos cirúrgicos pode prolongar a recuperação pós-operatória, aumentar a morbidade e a mortalidade e levar a um impacto financeiro desnecessário para a instituição. Espera-se que os resultados deste estudo possam motivar a equipe multiprofissional à realização da manutenção preventiva dos equipamentos antes das cirurgias. Palavras-chave: Centros cirúrgicos. Equipamentos cirúrgicos. Manutenção de equipamento.

ABSTRACT: Objective: To identify the implications of non-maintenance of hospital equipment for the quality of surgical care. Method: This is a quantitative, exploratory, descriptive, observational study carried out at a philanthropic hospital in the countryside of Minas Gerais. The technique of direct observation was applied, as well as the evaluation of records related to preventive and corrective maintenance of surgical equipment. Results: During the observation period, the equipment presenting most failures during surgical procedures were: electric scalpel, intensifier, and surgical focus. Equipment functionality and maintenance data were compared with manufacturers' recommendations and the scientific literature. Conclusion: Failure in surgical equipment maintenance can prolong patients' postoperative recovery, increase morbidity and mortality, and lead to unnecessary financial impact for the institution. It is hoped that the results of this study motivate the multiprofessional team to perform preventive maintenance of equipment before surgeries. Keywords: Surgical centers. Surgical equipment. Equipment maintenance.

RESUMEN: Objetivo: Identificar las implicaciones del no mantenimiento de los equipos hospitalarios en la calidad de la atención quirúrgica. Método: Se trata de un estudio observacional, descriptivo, exploratorio y cuantitativo realizado en un hospital filantrópico del interior de Minas Gerais. Se aplicó la técnica de observación directa y la evaluación de registros de mantenimiento preventivo y correctivo de los equipos quirúrgicos. Resultados: Durante el período de observación, se verificó que los equipos que presentaron el mayor número de fallas durante la cirugía fueron: bisturí eléctrico, intensificador y foco quirúrgico. La funcionalidad del equipo y los datos de mantenimiento se compararon con las recomendaciones del fabricante y la literatura científica. Conclusión: La falta de mantenimiento del equipo quirúrgico puede prolongar la recuperación postoperatoria, aumentar la morbilidad y la mortalidad y generar un impacto financiero innecesario para la institución. Se espera que los resultados de este estudio motiven al equipo multiprofesional a realizar el mantenimiento preventivo del equipo antes de las cirugías. Palabras clave: Centros quirúrgicos. Equipo quirúrgico. Mantenimiento de equipo.

\footnotetext{
'Enfermeira; doutora em Engenharia Mecânica; professora adjunta do Departamento de Enfermagem da Universidade Federal dos Vales do Jequitinhonha e Mucuri - Diamantina (MG), Brasil. 'Enfermeira; doutora em Engenharia Mecânica; professora adjunta do Departamento de Enfermagem

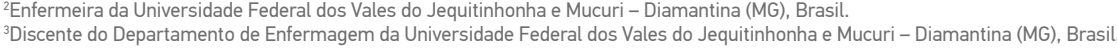

${ }^{4}$ Enfermeira mestre em Enfermagem Psiquiátrica; professora Adjunta do Departamento de Enfermagem da Universidade Federal dos Vales do Jequitinhonha e Mucuri - Diamantina (MG), Brasil.

*Autora correspondente: thabataclucas@gmail.com

Recebido: 28/11/2017 - Aprovado: 13/03/2018
}

DOI: $10.5327 / Z 1414-4425201800020003$ 


\section{INTRODUÇÃO}

Um dos desafios mundiais da Organização Mundial da Saúde (OMS) para a segurança do paciente cirúrgico inclui avaliar padrões mínimos de segurança de equipamentos pertencentes ao centro cirúrgico (CC), além dos itens mais significativos de risco à segurança do paciente ${ }^{1,2}$. Em 2009 e 2014, a OMS publicou Guidelines para cirurgias seguras, a fim de reduzir a ocorrência de eventos adversos ao paciente cirúrgico e definir padrões de segurança que podem ser aplicados a todos os países ${ }^{1,2}$.

Dentro desse contexto, alguns países da Europa, por exemplo, ainda não conseguem melhorar as taxas de eventos adversos nos CC das instituições hospitalares, o que confirma que o desafio implementado pela OMS é um problema persistente, não somente no Brasil, mas em nível mundial ${ }^{3}$.

No Brasil, estima-se que 3 a $16 \%$ dos pacientes hospitalizados sofrem eventos adversos e que mais da metade desses eventos podem ser evitados ${ }^{4}$. Há uma taxa de $3 \%$ de eventos adversos perioperatórios ${ }^{1}$. Além disso, dentre as cirurgias consideradas de alta complexidade, registra-se que cerca de $16 \%$ são realizadas em pacientes internados, com significativas taxas de óbito 5 .

A vigilância de eventos adversos é uma das práticas consideradas padrão ouro para melhorar a assistência ao paciente durante as cirurgias ${ }^{2,3}$. A avaliação da qualidade e da segurança dos equipamentos tem sido criticamente apontada como ponto-chave para o atendimento seguro do paciente. Apesar da emergente necessidade, poucos estudos têm sido desenvolvidos enfatizando a manutenção desta prática, sobretudo nos $\mathrm{CC}^{3-6}$.

Estudo recente aponta que, de acordo com a International Organization for Standardization (ISO), olaringoscópio, por exemplo, tem que estar com a medida mínima aceitável de brilho e luminosidade para ser utilizado em intubação no caso de cirurgias eletivas $^{6}$. Nesse estudo, realizado num hospital de Norfolk, Virgínia, Estados Unidos, apenas 29\% dos 283 laringoscópios avaliados estavam de acordo com o padronizado ${ }^{6}$. No entanto, nenhum evento adverso foi relatado com o uso desses equipamentos.

OCC é uma das unidades mais complexas do hospital; assim, devido às intervenções cirúrgicas e à acentuada transição de funcionários de diversas classes, tem maior predisposição para o acontecimento de eventos adversos ${ }^{5}$. As salas operatórias (SO) são formadas por equipamentos que devem estar funcionando corretamente para garantir a segurança do paciente, diminuindo o número de incidentes recorrentes no ambiente cirúrgico.

As SO dispõem de determinados equipamentos hospitalares, como bisturis e aparelhos de anestesia, que, apesar de permitirem tempo longo de manutenção, devem ser inspecionados diariamente para a simples detecção de oxidação dos cabos e ajuste de gases, por exemplo. Segundo a Association Française de Normalisation (AFNOR), manutenção é um conjunto de ações destinadas a garantir o bom funcionamento dos equipamentos, por meio de fiscalizações para medir seu desempenho, com o intuito de aumentar sua vida útil e proporcionar segurança ao paciente ${ }^{7}$. A manutenção preventiva é essencial para estender a vida produtiva do equipamento, com redução de custos e melhoria da segurança e do desempenho, porém os recursos financeiros têm restringido o desenvolvimento de programas para esse fim.

Para garantir que a inspeção seja adequada e feita periodicamente, é necessário que a instituição tenha um roteiro de manutenção, o que assegura um nível mínimo de inspeção de qualidade. A frequência com que a manutenção preventiva é feita varia de acordo com o preconizado pelo fabricante. Quando ocorre falha no equipamento, no entanto, é preciso acionar a manutenção corretiva, que consiste em reparar o defeito que o levou a parar de funcionar.

Cumpre destacar que a maioria das adversidades pode ser prevenida se houver gerenciamento correto dos técnicos de manutenção e uso adequado dos equipamentos pela equipe. A possibilidade de ocorrer algum evento durante a cirurgia poderá diminuir, porém a melhor forma de evitar esses eventos é fazendo o planejamento adequado da manutenção preventiva.

Diante da lacuna no conhecimento científico e das instituições hospitalares no que se refere aos eventos adversos provenientes de equipamentos no CC, vislumbra-se no presente artigo uma discussão crítica a respeito das manutenções e condições adequadas destes itens. Além disso, o presente artigo teve o objetivo de contribuir para a melhora do nível de segurança dos pacientes cirúrgicos, protegendo a comunidade de danos evitáveis e reduzindo eventos adversos em hospitais.

\section{OBJETIVO}

Identificar as implicações da não manutenção dos equipamentos hospitalares na qualidade do atendimento cirúrgico.

\section{MÉTODO}

Trata-se de um estudo de abordagem quantitativa, exploratório-descritivo, realizado no CC de um hospital filantrópico no interior de Minas Gerais. 
O hospital presta atendimento de urgência, emergência e internações. São realizadas cirurgias de pequeno, médio e grande porte, diagnósticos laboratoriais e de imagem. A unidade realiza, em média, de 150 a 200 cirurgias por mês. Utilizou-se a técnica de observação direta dos equipamentos do bloco cirúrgico, sendo que a equipe foi treinada de acordo com o manual de observadores proposto pela OMS e disponibilizado em português pela Agência Nacional de Vigilância Sanitária (ANVISA) e pela Organização Pan-Americana da Saúde (OPAS) $)^{1,2}$. As sessões de observação foram distribuídas nos turnos de trabalho matutino, vespertino e noturno. O tempo de observação foi compreendido num intervalo de 40 a 60 minutos pelo período de 7 meses. O período de observação foi de setembro de 2016 a março de 2017.

A coleta dos dados foi realizada por estudantes de graduação e de mestrado previamente treinados. $O$ treinamento fundamentou-se na leitura dos seguintes materiais: Guidelines for Recalls, Corrections and Removals (Devices) da Food and Drug Administration (FDA), Segundo desafio global para a segurança do paciente: Cirurgias seguras salvam vidas (orientações para cirurgia segura da OMS) e o documento de referência para o Programa Nacional de Segurança do Paciente (PNSP) do Ministério da Saúde (MS).

Os alunos foram liberados para coleta após atingir um nível de concordância de, no mínimo, $85 \%$ com o pesquisador principal. Os estudantes acompanharam cirurgias no dia da observação a fim de presenciar qualquer evento adverso causado por mau funcionamento dos equipamentos. Avaliaram-se os registros de manutenção preventiva e corretiva dos equipamentos cirúrgicos que havia na instituição.

Para a observação das cirurgias e dos equipamentos do CC, utilizou-se um formulário que continha as seguintes informações relacionadas à manutenção dos itens: mau funcionamento, calibração e ocorrência de eventos adversos provenientes dos equipamentos durante a cirurgia. Os dados de funcionalidade e deterioração dos equipamentos foram comparados com recomendações do fabricante e com a literatura científica. Os dados foram analisados por meio de análise comparativa dessas informações no programa Microsoft Excel ${ }^{\circledR}$.

\section{RESULTADOS}

De acordo com os dados coletados no processo de observação, o CC possui quatro SO, uma sala de pós-operatório, com três leitos adultos e um pediátrico, um lavatório para escovação das mãos, com três torneiras de pedal, uma sala para armazenamento dos materiais de cirurgia e um expurgo. As SO do CC contêm cinco focos cirúrgicos, sendo quatro afixados no teto e um portátil. Ressalta-se que o foco afixado no teto da sala 1 não estava funcionando há um período superior a 6 meses. $\mathrm{O}$ CC possui, ainda, oito monitores multiparâmetros, sendo que quatro ficam nas $\mathrm{SO}$ e quatro na sala de pós-operatório.

Durante o período de observação, verificou-se que os equipamentos que mais apresentaram falhas durante a cirurgia foram: foco, intensificador e bisturi elétrico. No período de observação deste estudo, presenciaram-se dez falhas no bisturi elétrico, sete falhas no intensificador e três falhas no foco cirúrgico durante as cirurgias (Figura 1). Não foi observado, no entanto, nenhum evento adverso com os pacientes decorrente da inadequação dos equipamentos.

O Quadro 1 apresenta os equipamentos observados e a comparação das datas de manutenção, como recomendado pelo fabricante e pela ANVISA.

\section{DISCUSSÃO}

No presente estudo, observou-se que havia equipamentos sem registros de manutenção no CC e um intensificador e foco cirúrgico que não estavam funcionando. Na prática hospitalar, observaram-se atraso e cancelamento de cirurgias decorrentes de intensificadores que apresentaram defeito durante o procedimento e da ausência de outros para repor. Aquele que estava funcionando apresentou falhas durante a cirurgia (Figura 1), e as cirurgias que necessitavam do equipamento foram realizadas em outra instituição, causando transtorno de deslocamento dos pacientes e funcionários do hospital.

Apesar de a recomendação do fabricante para manutenção de intensificadores ser a cada 6 meses, procedimentos simples diários poderiam evitar tais transtornos, como a verificação do funcionamento da bateria, da ventilação e do desempenho elétrico, como mostrado no Quadro 1.

Cumpre destacar que é essencial a criação de diretrizes de Procedimentos Operacionais Padrão (POPs) para direcionamento das manutenções, sobretudo devido às inúmeras recomendações dos fabricantes e à fragilidade dos equipamentos cirúrgicos, que pode provocar o seu não funcionamento no período de realização de uma cirurgia ${ }^{6,7}$.

É importante sensibilizar a equipe multiprofissional de que é necessário o entendimento das recomendações mais simples do fabricante para a utilização dos equipamentos na prática diária, como, por exemplo, verificação de oxidação nos cabos, pressões adequadas no manômetro e funcionamento de lâmpadas para evitar a ocorrência de eventos adversos aos pacientes. 


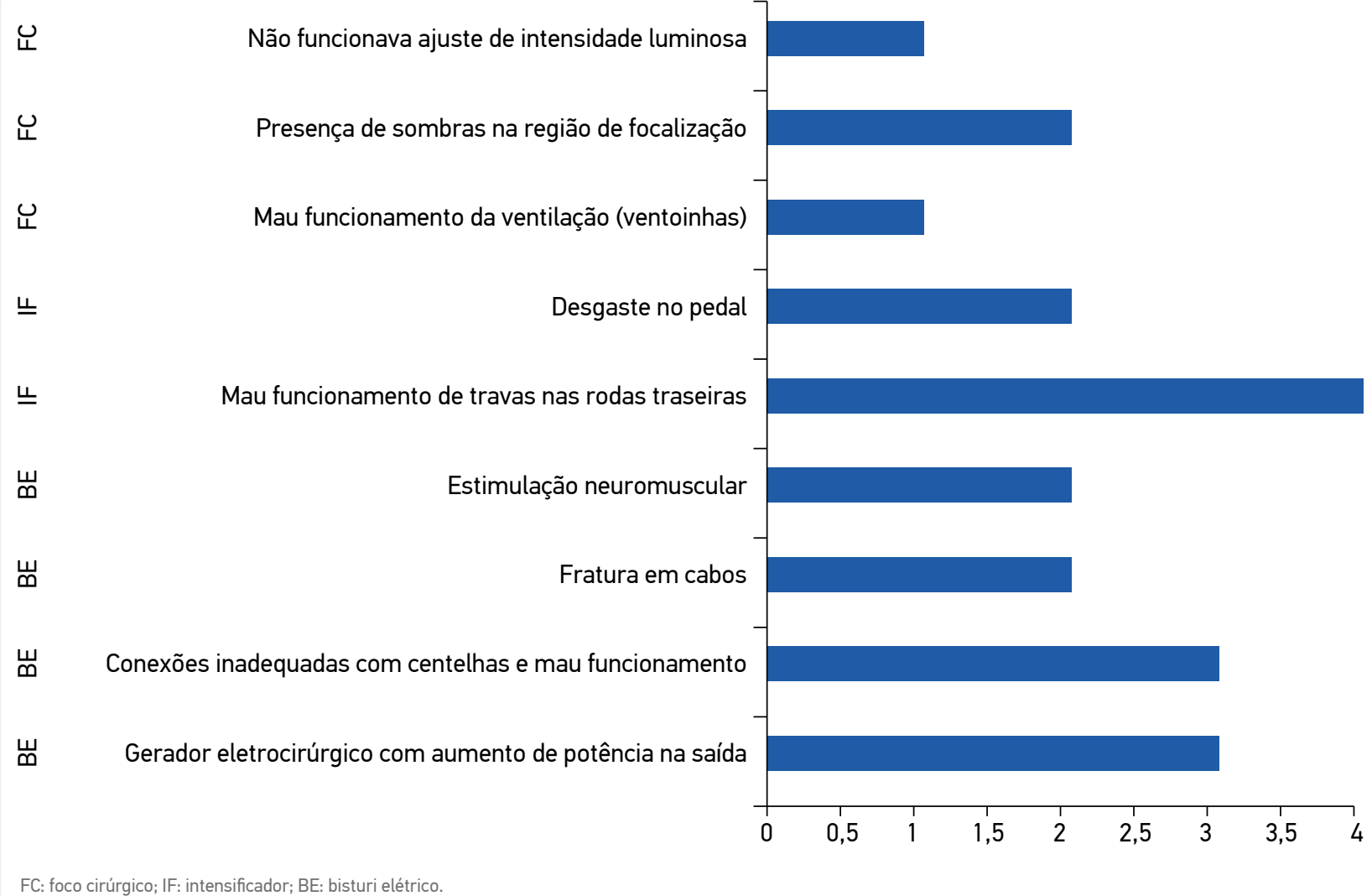

Figura 1. Distribuição das falhas observadas segundo o tipo de equipamento. Diamantina, Minas Gerais, 2017.

Quadro 1. Manutenção de equipamentos hospitalares, segundo as recomendações do fabricante e da Agência Nacional de Vigilância Sanitária. Diamantina, Minas Gerais, 2017.

\begin{tabular}{|c|c|c|c|c|}
\hline Equipamento & Especificação & Quantidade & $\begin{array}{l}\text { Data da última } \\
\text { manutenção }\end{array}$ & Preconizado pelo fabricante e pela ANVISA \\
\hline $\begin{array}{l}\text { Aparelhos de } \\
\text { anestesia }\end{array}$ & $\begin{array}{l}\text { Origami plus k Takaoka }{ }^{\circledR} \text {. } \\
\text { Ásia, América Latina } \\
\text { DrägerFabius }{ }^{\circledR} \text { Plus, Lübeck, } \\
\text { Alemanha }\end{array}$ & $\begin{array}{l}1 \\
3\end{array}$ & $\begin{array}{l}29 / 09 / 2015 \\
\text { Sem data de } \\
\text { registro }\end{array}$ & $\begin{array}{l}\text { Manutenção preventiva: a cada } 12 \text { meses. } \\
\text { A cada cirurgia avaliar: } \\
\text { - conexões do equipamento; } \\
\text { - funcionamento do sistema de exaustão; } \\
\text { - ajuste de fluxo de gás; } \\
\text { - aspirador; } \\
\text { - mangueiras; } \\
\text { - pressão de gases. }\end{array}$ \\
\hline $\begin{array}{l}\text { Aspirador } \\
\text { portátil }\end{array}$ & $\begin{array}{l}\text { Dia-Pump }{ }^{\circledR} \text { Fanem, 089/ } \\
\text { R2D2. São Paulo, Brasil }\end{array}$ & 1 & $18 / 12 / 2013$ & $\begin{array}{l}\text { Manutenção preventiva: a cada seis meses. } \\
\text { A cada três meses: } \\
\text { - trocar o microfiltro; } \\
\text { - realizar limpeza do microventilador. } \\
\text { Verificar diariamente: display, placa de circuito, } \\
\text { manômetro, regulagem da bomba pressurizadora } \\
\text { e higienização. }\end{array}$ \\
\hline $\begin{array}{l}\text { Bisturis } \\
\text { elétricos }\end{array}$ & $\begin{array}{l}\text { Wem }^{\circledR}, \text { SS-501s. Bruxelas, } \\
\text { União Europeia } \\
\text { Wem }^{\circledR}, \text { SS-501s. Bruxelas, } \\
\text { União Europeia } \\
\text { Deltronix }{ }^{\circledR} \text {. Ribeirão Preto, } \\
\quad \text { São Paulo, Brasil }\end{array}$ & $\begin{array}{l}2 \\
1 \\
1\end{array}$ & $\begin{array}{l}\text { Sem data de } \\
\text { registro } \\
11 / 12 / 2015 \\
16 / 05 / 2013\end{array}$ & $\begin{array}{l}\text { Manutenção preventiva: pelo menos uma vez ao ano. } \\
\text { Verificar diariamente: existência de oxidação nos } \\
\text { cabos de energia e presença de danos físicos na } \\
\text { carcaça do equipamento. } \\
\text { Semanalmente: verificar condições da fonte de energia. }\end{array}$ \\
\hline
\end{tabular}


Quadro 1. Continuação.

\begin{tabular}{|c|c|c|c|c|}
\hline Equipamento & Especificação & Quantidade & $\begin{array}{c}\text { Data da última } \\
\text { manutenção }\end{array}$ & Preconizado pelo fabricante e pela ANVISA \\
\hline Cardioversor & $\begin{array}{l}\text { Medtronic Lifepak }{ }^{\circledR} 20 \text {, } \\
\text { Tolochenaz, Suíça }\end{array}$ & 1 & $21 / 03 / 2013$ & $\begin{array}{l}\text { Manutenção preventiva: pelo menos uma vez ao ano. } \\
\text { Verificar diariamente: carga de bateria e descarga } \\
\text { aplicada em um analisador de desfibriladores. } \\
\text { A cada seis meses: } \\
\text { - verificar DEA; } \\
\text { - avaliar condições de desfibrilação das pás padrão; } \\
\text { - avaliar o marcapasso; } \\
\text { - avaliar as condições de cardioversão } \\
\text { sincronizada do cabo de terapia. }\end{array}$ \\
\hline $\begin{array}{l}\text { Focos } \\
\text { cirúrgicos }\end{array}$ & $\begin{array}{l}\text { Hanaulux }{ }^{\circledast} \text { Blue } 80 \text {, Renânia } \\
\text { do Norte-Vestefália, } \\
\text { Alemanha } \\
\text { Hanaulux } \\
\text { do Blue } 80 \text {, Renânia } \\
\text { Alemanha } \\
\text { Baumeria, } \\
\text { Pa Mogi Mirim, São } \\
\text { Paulo, Brasil }\end{array}$ & $\begin{array}{l}1 \\
3 \\
1\end{array}$ & $\begin{array}{l}\text { Sem data de } \\
\text { registro (não } \\
\text { funcionava para } \\
\text { uso) } \\
\text { Sem data de } \\
\text { registro } \\
18 / 07 / 2013\end{array}$ & $\begin{array}{l}\text { Manutenção preventiva: não deve exceder dois anos. } \\
\text { Semanalmente: } \\
\text { - fazer a inspeção funcional e visual do foco cirúrgico; } \\
\text { - avaliar o estado de conservação da carcaça; } \\
\text { - avaliar o amortecedor dos focos; } \\
\text { - avaliar se as cúpulas estão em posição adequada; } \\
\text { - inspecionar se as lâmpadas estão centradas e fixas; } \\
\text { - verificar a segurança elétrica do sistema de iluminação. }\end{array}$ \\
\hline $\begin{array}{l}\text { Monitores } \\
\text { multiparâmetros }\end{array}$ & $\begin{array}{l}\text { Dixtal Biomédica Ind e Com. } \\
\text { Ltda, dx 2023. Manaus, } \\
\text { Amazonas, Brasil } \\
\text { Dixtal Biomédica Ind. e Com. } \\
\text { Ltda, dx 2023. Manaus, } \\
\text { Amazonas, Brasil }\end{array}$ & $\begin{array}{l}1 \\
7\end{array}$ & $\begin{array}{c}30 / 11 / 2012 \\
\text { Sem data de } \\
\text { registro }\end{array}$ & $\begin{array}{l}\text { Manutenção preventiva: anualmente. } \\
\text { Deve-se avaliar: } \\
\text { - calibração; } \\
\text { - medida de isolamento elétrico do aparelho; } \\
\text { - controles elétricos, parafusos e indicadores } \\
\text { sonoros e visuais. } \\
\text { A cada três meses verificar: } \\
\text { - presença de ressecamento de borrachas e } \\
\text { conexões; } \\
\text { - trincamento de partes plásticas e conectores; } \\
\text { - oxidação de partes metálicas; } \\
\text { - rompimento de cabos; } \\
\text { - falhas no alarme sonoro ou visual. }\end{array}$ \\
\hline Intensificadores & $\begin{array}{l}\text { OEC Fluorostar } 7900 . \\
\text { General Electric Company } \\
\text { Buc, França } \\
\text { Opescope Activo Shimadzu } \\
\text { Corporation Ltda. Kyoto, } \\
\text { Japão }\end{array}$ & $\begin{array}{l}1 \\
1\end{array}$ & $\begin{array}{c}\text { Sem data de } \\
\text { registro (não } \\
\text { funcionava para } \\
\text { uso) } \\
14 / 12 / 2016\end{array}$ & $\begin{array}{l}\text { Manutenção preventiva: a cada seis meses. } \\
\text { Deve-se avaliar: } \\
\text { - movimento manual das travas e componentes } \\
\text { mecânicos; } \\
\text { - desempenho eletromecânico; } \\
\text { - desempenho do bloqueio de segurança; } \\
\text { - desempenho elétrico, } \\
\text { - funcionamento da bateria e descarga } \\
\text { eletrostática; } \\
\text { - funcionamento da ventilação; } \\
\text { - funcionamento da geração e resolução de imagens. }\end{array}$ \\
\hline $\begin{array}{l}\text { Laringoscópio } \\
\text { de fibra óptica }\end{array}$ & $\begin{array}{l}\text { M/S SNAA Industries, } \\
\text { Paquistão }\end{array}$ & 4 & $\begin{array}{l}\text { Sem data de } \\
\text { registro }\end{array}$ & $\begin{array}{l}\text { Manutenção preventiva: antes de cada utilização. } \\
\text { Deve-se avaliar: } \\
\text { - integridade das pilhas; } \\
\text { - correto funcionamento da lâmpada; } \\
\text { - itens deteriorados devem ser substituídos } \\
\text { sempre que necessário. }\end{array}$ \\
\hline $\begin{array}{l}\text { Mesas } \\
\text { cirúrgicas }\end{array}$ & $\begin{array}{c}\text { Mercedes IMEC }{ }^{\circledast} \text {. São Paulo, } \\
\text { Brasil }\end{array}$ & 4 & $\begin{array}{l}\text { Sem data de } \\
\text { registro }\end{array}$ & $\begin{array}{l}\text { Manutenção preventiva: pelo menos uma vez ao ano. } \\
\text { Deve-se verificar: } \\
\text { - partes elétricas; } \\
\text { - alinhamento; } \\
\text { - lubrificação; } \\
\text { - desgaste; } \\
\text { - limpeza. }\end{array}$ \\
\hline
\end{tabular}

Anvisa: Agência Nacional de Vigilância Sanitária; DEA: desfibrilador externo automático. 
A manutenção tem como finalidade garantir o funcionamento adequado dos equipamentos e instalações, assegurando um serviço de qualidade para manter a vida útil do aparelho, tendo um valor apropriado para a tarefa exercida ${ }^{3}$.

No presente estudo, observou-se que alguns equipamentos, como bisturis elétricos, aspirador portátil, cardioversor e monitores multiparâmetros, possuíam a data de manutenção com registro desatualizado. Tal fato pode sugerir que, frequentemente, os gestores ou a equipe multiprofissional esperam que aconteça algum problema nos equipamentos para acionar a manutenção corretiva, o que pode gerar atraso de cirurgias, não funcionamento dos aparelhos e eventos adversos nos pacientes que, muitas vezes, já se encontram na mesa de cirurgia.

A Associação Brasileira de Normas Técnicas (ABNT) considera que manutenção é "a combinação de todas as ações técnicas e administrativas destinadas a manter ou recolocar um item em um estado no qual possa desempenhar uma função requerida”.".

Um estudo realizado em um hospital de ensino na região Centro-Sul do Estado de São Paulo apontou que 31,9\% dos eventos adversos informados estavam diretamente relacionados aos equipamentos cirúrgicos ${ }^{4}$.

Outro estudo, feito em um hospital de ensino da região Centro-Oeste do Brasil, apontou a ocorrência de 42 episódios de eventos adversos, dos quais $26,2 \%$ associavam-se a problemas de estrutura das SO, como manutenção dos equipamentos e abastecimento de materiais ${ }^{5}$. A maioria $(73,8 \%)$ dos eventos adversos foram causados por problemas com o aparelho de anestesia e queimadura pelo uso de bisturi elétrico ${ }^{5}$.

No que se refere aos bisturis elétricos, é necessário observar a presença de oxidação nos cabos de energia, danos físicos na carcaça do equipamento e as condições da fonte de energia, a fim de evitar a ocorrência de eventos adversos. Isso demonstra como a verificação do bisturi elétrico previamente à cirurgia pelos cirurgiões e instrumentadores, principalmente por estarem de luva estéril, possibilita não torná-los suscetíveis a danos aos pacientes que se encontram em intervenções cirúrgicas.

Quanto aos focos cirúrgicos observados neste estudo, verificou-se que apresentavam sombras, o que dificultava a luminosidade ideal para a realização da cirurgia. Uma inspeção visual diária é necessária, pois os requisitos mínimos dos focos devem ser cumpridos para que a cirurgia seja realizada com mais segurança.

Na presente pesquisa, três aparelhos de anestesia não apresentavam registro de manutenção. Sabe-se que complicações com aparelhos de anestesia permanecem como uma causa substancial de morte em CC, em nível global ${ }^{8,9}$. A persistência desse dado poderá ser revertida, caso a equipe multiprofissional fique atenta para a importância das avaliações diárias nesses equipamentos.

Quanto aos laringoscópios, a avaliação diária é essencial para atender o paciente de forma segura na intubação traqueal e na anestesia. A manutenção dos laringoscópios envolve, sobretudo, qualidade e níveis aceitáveis de luz para o procedimento 6 . Um estudo realizado em Norfolk (Virgínia, Estados Unidos) avaliou a qualidade da iluminação de 691 laringoscópios, dos quais $28 \%$ estavam abaixo do padrão preconizado (entre 500 e 867 lux $)^{6}$. Além disso, testes de medida de luz nunca haviam sido realizados em nenhum dos laringoscópios. Em vez disso, eram feitas apenas inspeções qualitativas visuais, que não garantiam a qualidade na eficiência dos equipamentos ${ }^{6}$.

A negligência na realização de testes físicos e mecânicos não pode acontecer nas instituições de saúde, que visam a qualidade do atendimento ao paciente, apesar de a inspeção visual ajudar a visualizar desgastes, deterioração e oxidação em alguns equipamentos.

Os suportes e o cilindro dos gases pertencentes às $\mathrm{SO}$, observados neste trabalho, apresentavam ferrugem disseminada por toda sua extensão, dificultando a visualização das informações de padronização, e, ainda, havia alguns sem lacre. Dessa forma, pensando na prevenção desses incidentes, a instituição deve elaborar e disseminar POPs, rotinas, guidelines, bundles, entre outros, para que haja padronização de técnicas, procedimentos e condutas, aumentando a segurança do paciente e do profissional, de modo a evitar a ocorrência de eventos adversos.

Sabe-se que mais da metade dos eventos adversos com equipamentos cirúrgicos podem ser evitados com a manutenção em dia e a verificação de tais aparelhos antes de cada cirurgia pela própria equipe multiprofissional ${ }^{6-11}$. Apesar da ocorrência de eventos adversos durante intervenções cirúrgicas ser reconhecida como um problema de saúde pública, tal problema ainda não é reconhecido pelas instituições de saúde ${ }^{6-11}$. Isso se deve a deficiências na gestão organizacional, econômica e financeira das instituições, sobretudo nos países em desenvolvimento.

Em 2017, a FDA levantou questionamentos em um hospital no estado de Michigan (Estados Unidos), em que equipamentos cirúrgicos apresentaram defeitos durante cirurgias devido à manutenção inadequada, colocando em risco a vida dos pacientes ${ }^{8}$. Um estudo observacional, realizado na Inglaterra e no País de Gales, divulgou que $6 \%(n=754)$ dos pacientes cirúrgicos apresentaram eventos adversos causados pela não manutenção ou manutenção inadequada dos equipamentos hospitalares ${ }^{8}$. Esse mesmo estudo verificou que $12 \%(\mathrm{n}=116)$ dos pacientes que sofreram eventos adversos provenientes de equipamentos ficaram gravemente feridos 
ou morreram ${ }^{7}$. A maioria das instituições de saúde, no entanto, não divulga dados quantitativos desses eventos.

As instituições de saúde deveriam ser encorajadas a desenvolver a cultura de registrar incidentes cirúrgicos causados por equipamentos, o que contribuiria para a criação de um banco de dados associado aos eventos adversos. Isso exigiria o comprometimento da gestão e da equipe multiprofissional de saúde, a fim de realizar uma vigilância minuciosa diária. Por outro lado, levaria a potenciais soluções de segurança das cirurgias com dados registrados e validados que poderiam ser aplicados na prática hospitalar.

Cumpre destacar que os termos falência do dispositivo ou equipamento médico são utilizados pela FDA para regulamentar o rastreamento da manutenção da qualidade do material ${ }^{8}$. Tais termos denotam a falha de determinado material em realizar a sua função, incluindo qualquer desvio ou anormalidade das especificações de performance ou uso pretendido ${ }^{10,11}$. Dessa forma, percebe-se a importância da produção de dados consistentes para a obtenção de requisitos mínimos de qualidade que favoreçam a vida útil dos equipamentos cirúrgicos.

Entretanto, não obstante a proposta desafiadora de manutenção preventiva e corretiva, seja por questões institucionais, econômicas ou legais, muitas instituições hospitalares não se dedicam à construção de protocolos que atendam aos parâmetros mínimos de qualidade desses equipamentos.

Os resultados deste estudo demonstram ausência de manutenção preventiva e tempo prolongado para a realização da manutenção corretiva dos equipamentos hospitalares. Assim, os reparos são realizados somente quando não há condições de uso dos aparelhos, que passam, então, a necessitar de maior tempo para correção, influenciando diretamente na qualidade da assistência prestada.

Uma limitação deste estudo foi a não realização de testes experimentais nos equipamentos que apresentaram falhas, pois os testes validam e comprovam o desgaste e o mau funcionamento, sobretudo no gerador eletrocirúrgico de bisturis elétricos, que, como consequência, podem levar a eventos adversos, como queimaduras em sítios e tecidos cirúrgicos.

Os achados deste estudo, no entanto, motivam a equipe multiprofissional à realização da manutenção preventiva e vigilância aos equipamentos hospitalares antes das cirurgias. Finalmente, a inadequada manutenção preventiva e corretiva dos equipamentos pode prolongar a recuperação pós-operatória, aumentar a morbimortalidade e gerar um aumento do impacto financeiro institucional ${ }^{10,11}$. Além disso, as normas de segurança e atendimento de qualidade fazem parte de um conjunto de requisitos necessários para o programa de segurança do paciente cirúrgico em nível hospitalar.

\section{CONSIDERAÇÕES FINAIS}

Conclui-se que a manutenção dos equipamentos hospitalares é essencial, seja para fornecer às instituições mecanismos de segurança do paciente cirúrgico, seja para iniciar um programa de segurança das cirurgias na prevenção de eventos adversos.

O CC, por ser um setor de alta complexidade, deve promover uma avaliação diária previamente à realização de cirurgias. Os equipamentos devem ser testados quanto ao seu estado e funcionalidade, para evitar incidentes durante os procedimentos. Nesse sentido, mais estudos devem ser feitos a fim de elucidar o ideal cronograma de manutenção corretiva e preventiva dos equipamentos cirúrgicos.

A falta de registros e a precariedade da manutenção dos equipamentos da instituição-sede desta pesquisa indicam que ainda é um desafio para as instituições de saúde a prevenção adequada da manutenção de aparelhos, de forma a evitar eventos adversos nos pacientes cirúrgicos. Dessa forma, além de atender aos objetivos de uma pesquisa que visa a expansão e contribuição ao conhecimento existente, fundamentando transformações sociais que possam repercutir em uma reflexão, este estudo contribui para a proposição de indicadores de qualidade que possibilitem a implementação de protocolos validados e controle.

\section{REFERÊNCIAS}

1. Organização Mundial de Saúde. Segundo desafio global para a segurança do paciente. Cirurgias seguras salvam vidas. Orientações para cirurgia segura da Organização Mundial de Saúde. Rio de Janeiro: Organização Pan-Americana da Saúde; Ministério da Saúde; Agencia Nacional de Vigilância Sanitária; 2009.

2. Brasil. Ministério da Saúde. Documento de referência para o Programa Nacional de Segurança do Paciente. Ministério da Saúde; Fundação Oswaldo Cruz; Agência Nacional de Vigilância Sanitária. Brasília: Ministério da Saúde; 2014.
3. Heideveld-Chevalking AJ, Calsbeek H, Damen J, Gooszen H, Wolff AP. The impact of a standardized incident reporting system in the perioperative setting: a single center experience on 2,563 'nearmisses' and adverse events. Patient Safety Surgery [Internet]. 2014 [citado 3 mar. 2017];8(46):1-10. DOI: 10.1186/s13037-014-0046-1

4. Santana HT, Siqueira HN, Costa MMM, Oliveira DCAN, Gomes SM, Sousa FC, et al. A segurança do paciente cirúrgico na perspectiva da vigilância sanitária: uma reflexão teórica. Vigil Sanit Debate [Internet]. 2014 [citado 10 jan. 2017];2(2):34-42. DOI: 10.3395/vd.v2i2.124 
5. Bezerra WR, Bezerra ALQ, Paranaguá TTB, Bernardes MJC, Teixeira CC. Ocorrência de incidentes em um centro cirúrgico: estudo documental. Rev Eletr Enf [Internet]. 2015 [citado 10 jan. 2017];17(4):3-11. Disponível em: http://dx.doi.org/10.5216/ree. v17i4.33339

6. Volsky PG, Murphy MK, Darrow DH. Laryngoscope illuminance in a tertiary children's hospital: implications for quality laryngoscopy. JAMA Otolaryngol Head Neck Surg [Internet]. 2014 [citado 10 mar. 2017];140(7):603-7. DOI: 10.1001/jamaoto.2014.676

7. Carson-Stevens A, Hibbert P, Williams H, Evans HP, Cooper A, Rees P, et al. Characterising then nature of primary care patient safety incident reports in the England and Wales National Reporting and Learning System: a mixed-methods agenda-setting study for general practice. Health Serv Deliv Res [Internet]. 2016 [citado 10 mar. 2017];4(27). DOI: $10.3310 /$ hsdr04270
8. Food and Drug Administration. Guidelines for recalls, corrections and removals (devices) [lnternet]. 2017 [citado 3 mar. 2017]. Disponível em: https://www.fda.gov/MedicalDevices/DeviceRegulationandGuidance/ PostmarketRequirements/RecallsCorrectionsAndRemovals/default.htm

9. Murphy KM, Volsky PG, Darrow, DH. Laryngoscope illuminance in a tertiary care medical center: industry standards and implications for quality laryngoscopy. JAMA Otolaryngol Head Neck Surg [lnternet]. 2015 [citado 10 mar. 2017];153(5):806-11. DOI: 10.1177/0194599815587700

10. World Health Organization. International consultation on European validation of the minimal information model for patient safety incident reporting and learning. Technical Report. Warsaw, Poland: World Health Organization;2015.

11. Mull JH, Brennan CW, Folkes T, Hermos J, Chan J, Rosen AK, et al. Identifying previously undetected harm: piloting the institute for healthcare improvement's global trigger tool in the veterans health administration. Qual Manag Health Care [Internet]. 2015 [citado 10 mar. 2017];24(3):140-6. DOI: 10.1097/QMH.0000000000000060 